\title{
Dentin Dysplasia Type I: Report of Two Cases
}

\author{
'Zahra Elmi R, ${ }^{2}$ Somaye Azimi, ${ }^{3}$ Soudeh Jafari, ${ }^{4}$ Amir Farzanegan
}

\begin{abstract}
Objectives: In this article, we report two cases of type I dentin dysplasia (DD): the first case is subtype "d" DD and second case is severe form of DD type I, which presents all of four subtypes and describes characteristics and the management of this type of teeth anomalies.
\end{abstract}

Materials and methods: Dentin dysplasia is a rare abnormality of dentin that involves both primary and permanent dentition. The normal shape of crown, short roots or its total absence, pulp chamber obliteration, crescent-shaped pulp, pulp stone, and periapical radiolucencies are the characteristics of DD type I.

Conclusion: There are many problems in the management of a patient with DD. Depending on root development and pulp canal situation, endodontic treatment and tooth extraction are applied in teeth with pulp necrosis and periapical abscess. The prognosis of DD largely depends upon the age at which the diagnosis is made, and the quality of the treatment provided.

Keywords: Dentin dysplasia, Pulp obliteration, Rootless teeth. How to cite this article: Elmi R Z, Azimi S, Jafari S, Farzanegan A. Dentin Dysplasia Type I: Report of Two Cases. Int J Experiment Dent Sci 2017;6(1):44-47.

Source of support: Nil

Conflict of interest: None

\section{INTRODUCTION}

Dentin dysplasia (DD), the autosomal dominant hereditary disturbance in dentin formation, is a rare anomaly with unknown etiology, which occurs approximately 1 in every 100,000 in the world. ${ }^{1,2}$

The first case reported with this condition was in 1920 termed as "rootless teeth", and in 1939, Rushton termed this condition as DD. ${ }^{3}$ Dentinal dysplasia is classified into radicular dentin dysplasia (DDI) and coronal dysplasia (DDII). Both types include generalized/ multiple involvements of the primary and permanent

\footnotetext{
${ }^{1-3}$ Assistant Professor, ${ }^{4}$ Postgraduate Student

${ }^{1}$ Department of Oral and Maxillofacial Medicine, School of Dentistry, Guilan University of Medical Sciences, Rasht, Islamic Republic of Iran

${ }^{2,3}$ Department of Oral Medicine, Shahid Beheshti University of Medical Sciences, Tehran, Islamic Republic of Iran

${ }^{4}$ Department of Periodontics, Yazd University of Medical Sciences, School of Dentistry, Yazd, Islamic Republic of Iran

Corresponding Author: Amir Farzanegan, Postgraduate Student Department of Periodontics, Yazd University of Medical Sciences School of Dentistry, Yazd, Islamic Republic of Iran, e-mail: amirfarzanegan88@gmail.com
}

dentitions. The DD type I is more common. The DDI also called "rootless teeth" is characterized by the presence of teeth with normal appearing crowns, but no or only rudimentary root development, incomplete or total obliteration of the pulp chamber, and periapical radiolucent areas or cysts. In this anomaly, teeth may exhibit extreme mobility and exfoliate prematurely.,

The DDII, called coronal DD, is characterized by partial pulpal obliteration with the amorphous dentin often forming pulp stones, thistle-tube-shaped coronal pulp chambers, thread-like root canals, and the absence of periapical radiolucencies. Primary teeth with complete pulp obliteration and brown or amber bluish coloration are observed similar to that seen in hereditary opalescent dentin. The permanent teeth have a normal appearance or a slight amber coloration; the roots are normal in size and shape. ${ }^{4,6}$

This report describes two cases of DDI, clinical and radiographic view of this abnormality in detail.

\section{CASE REPORTS}

\section{Case 1}

A 24-year-old male was referred to the Oral Medicine Department of Shahid Beheshti University, Faculty of Dentistry, Tehran, for routine oral examination. The patient's medical history showed no remarkable problem but in habitual history, he had bruxism and mouth breathing. The profile was concave, the face had long appearance, and in the extraoral examination, the lower face had asymmetry. Intraoral examination showed severe maxillary constriction, class III occlusion, and crowding in anterior teeth of maxilla and mandible. The crowns had normal appearance (Fig. 1). The patient's oral hygiene was moderate. There was gingivitis, amalgam and composite fillings, and prosthetic restoration.

Panoramic view showed normal coronal enamel and dentin, normal pulp chamber size, but short and malformed roots prominently in molars and premolars. Periapical radiolucency of maxillary second premolar and left first molar with superficial filling was seen (Fig. 2). Another periapical radiolucency like left lateral incisor, right second premolar, and first molars had the same condition, which seemed related to the bad fillings or incomplete root canal therapy. However, the clinical and radiographic pattern of dentition defined DD type I as an appropriate diagnosis. 

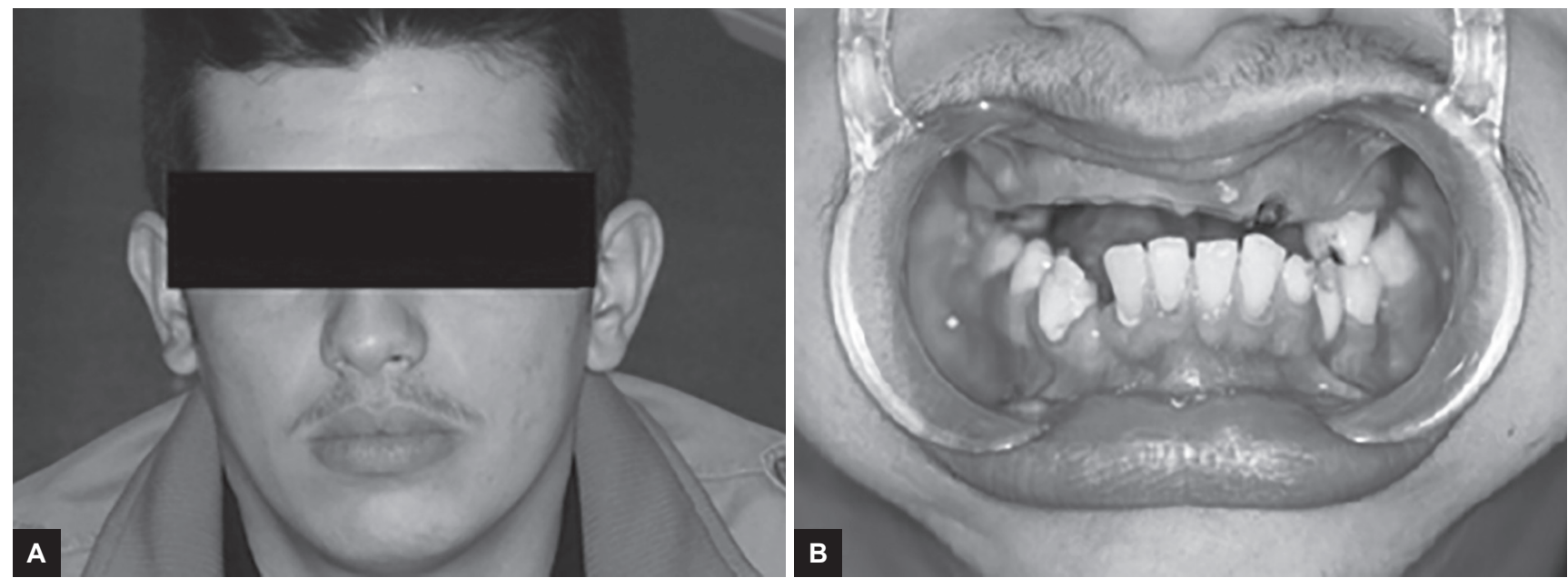

Figs 1A and B: (A) Patient's profile; and (B) severe crowding and maxillary constriction

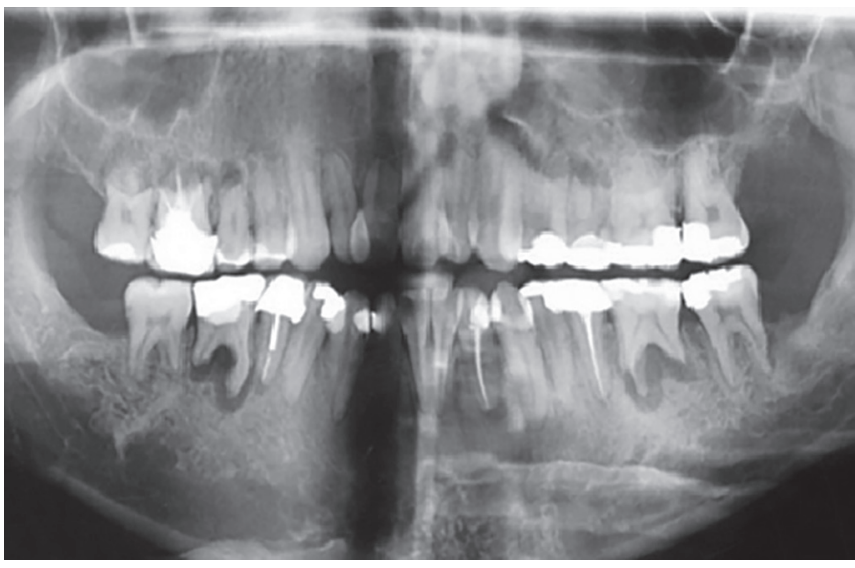

Fig. 2: Panoramic view of multiple periapical radiolucency and shortening of the roots, especially in the posterior teeth

The patient had no complaint about profile pattern of occlusion. Treatment plan consisted of determination of periodontal prognosis for lower first molars, endodontic treatment for teeth with periapical radiolucency, instructing oral hygiene, scaling, and root planing.

\section{Case 2}

An 18-year-old man attended the Oral Medicine Department of Shahid Beheshti Dental School, Tehran, with a complaint of teeth mobility and premature exfoliation of the teeth. Medical history was unremarkable.

Extraoral examination showed no abnormality, competent lips, and normal profile. Intraoral examination revealed the absence of most upper teeth except two molars and one premolar, and some remaining roots. Most of the lower teeth were present (Fig. 3).

On examination, molars and lower incisors show grade II mobility and premolars have grade III mobility except the lower right second premolar. The patient's oral hygiene was poor and all posterior remaining teeth had caries or filling. Plaque and calculus were present.

Radiographic examinations revealed teeth with very short, blunt, and malformed roots. Rudimentary root formation in upper and lower anterior teeth and molars was seen and all premolars except the lower
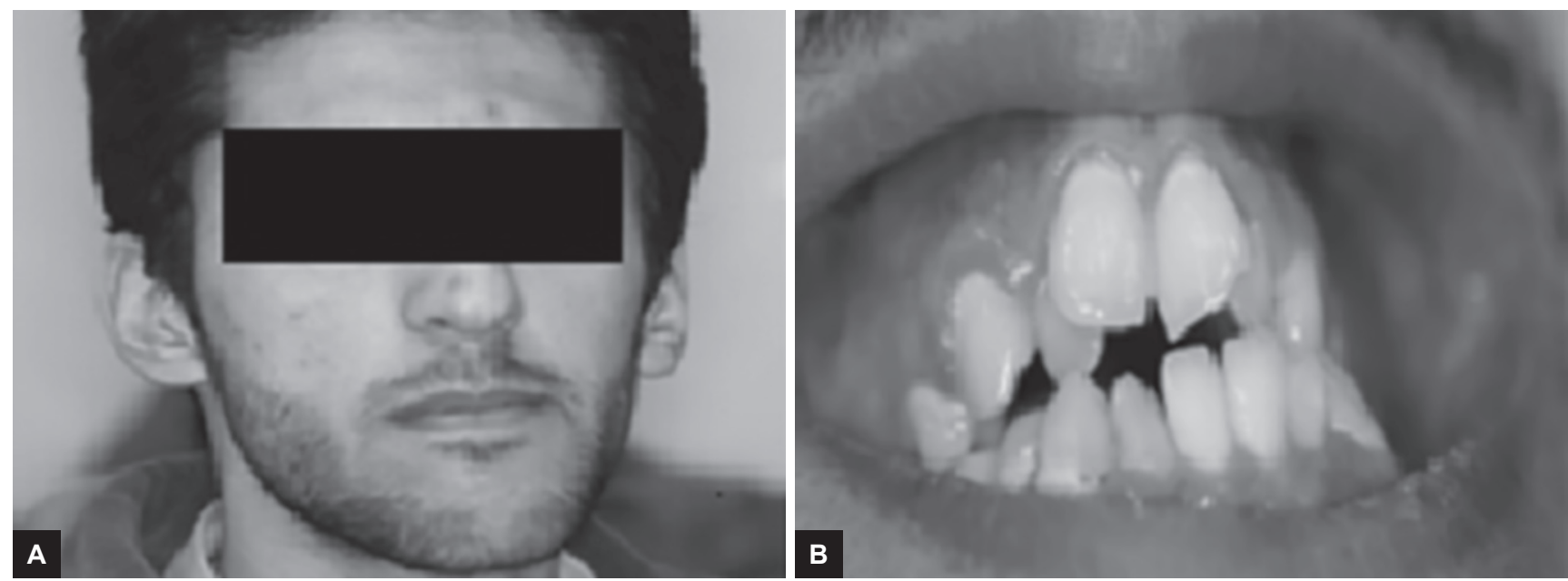

Figs 3A and B: (A) Normal profile; and (B) most of the teeth lost in the upper jaw 


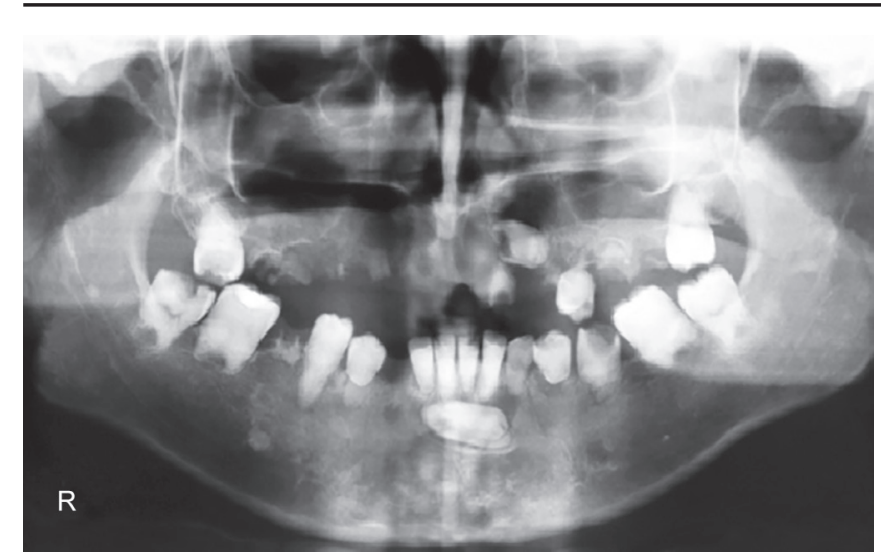

Fig. 4: Panoramic view shows the loss of a large number of teeth, too short roots, and absence of root. Obliterated pulp and crescentshaped pulp chamber

right second premolar showed no root formation or malformed roots (Fig. 4).

There was an impacted malformed canine. Lower primary canine in the left side still remained. All teeth showed pulp chamber obliteration. Crescent-shaped pulp chamber in the lower third molars was seen, which is characteristic of DDI.

Furthermore, radiographic findings showed periapical radiolucencies and many remaining roots in the jaws.

There was no family history of similar dental abnormality and also finding did not support the presence of systemic disorders.

Treatment strategies consisted of patient's information to improve oral health, extraction of all the mobile and periodontally hopeless teeth, and remaining roots. Root canal therapy due to pulp chamber obliteration was not feasible.

In order for mastication and esthetics, a provisional treatment was considered. Implant-supported prosthesis considering bone graft was advised as a final treatment.

\section{DISCUSSION}

The DD is a genetic defect of dentin formation, although its etiology is still considered a mystery. ${ }^{1}$ Wesley et $\mathrm{al}^{7}$ proposed that the condition is caused by an abnormal interaction of odontoblasts with ameloblasts, leading to abnormal differentiation and/or function of the ectomesenchymal-derived odontoblasts. The DD is usually an autosomal dominant condition, and there is a $50 \%$ chance that a child born to an affected parent will itself be affected. ${ }^{1}$

A DDI is also called radicular DD, primarily affecting the root's portions of the teeth and producing teeth with shortened roots and periapical radiolucencies. ${ }^{8}$

Type II DD also called coronal DD is characterized by large coronal pulp chamber containing denticles in permanent teeth and total pulp chamber obliteration in primary teeth. ${ }^{8}$

OCarroll categorized DDI as having four radiographic variations in permanent teeth. The variations are based on the amount of obliteration of the pulp chambers, root development, and periapical radiolucent areas. In the first category, DDIa, there is the complete obliteration of the pulp, no root development, and many periapical radiolucent areas; DDIb and DDIc have less pulpal obliteration and feature crescent-shaped radiolucent areas in the chamber, more root development, and less frequent periapical radiolucencies. In DDId, the pulp chambers are distinct, with pulp stones in the coronal third of the root canal (with corresponding bulging of the root); the root has significant development, and there are few, if any, periapical radiolucencies. 79

By this categorization, case 1 is placed in subtype DDId, and case 2 contains all subtypes of DDI. The radiographic characteristics of the permanent dentition can be used to differentiate DDI from DDII. ${ }^{9}$ Teeth with radiographic or histologic features of DD occur in a number of disorders, such as calcinosis, osteogenesis imperfecta, Ehlers-Danlos syndrome, Goldblatt syndrome, Schimke immuno-osseous dysplasia, and Brachio-skeletal-genital syndrome. $^{8}$

Azma et $\mathrm{al}^{10}$ report a case of DD type II associated with hypoplastic amelogenesis imperfecta in an 18-yearold girl, in Rasht, Guilan, without any syndromic signs. In explaining this coincidence, some studies have proposed that DD and dentinogenesis imperfecta represent allelic mutations of a common gene.

Toomarian et $\mathrm{al}^{11}$ present a case of DD type I in a 12-year-old Iranian boy. Radiographic examination revealed pulpless teeth with no root formation in most teeth. There was a radicular cyst in association with a permanent molar.

Shokri et $\mathrm{al}^{12}$ in 2014 performed a cross-sectional study on 1649 people in Iran, Hamadan, to evaluate the dental anomalies among 7- to 35-year-old patients using panoramic radiographs. The frequency of DD was 1 in 1,649 people with a prevalence of $0.06 \%$.

The management of patients with DD has presented dentists with many problems. Extraction has been suggested as an alternative treatment for teeth with pulp necrosis and periapical abscess. However, Ravanshad and $\mathrm{Khayat}^{13}$ reported conventional endodontic treatment for cases of DDI with pulp necrosis and periapical radiolucencies in a 22-year-old woman whose endodontically treated teeth were asymptomatic at 3 and 6 months and from 1 to 4 years after treatment in 2006. Radiographic examination showed complete resolution of the periapical lesions. 
Endodontic treatment is contraindicated in teeth with total obliteration of root canals and pulp chambers similar to our case 2 report.

Ansari et $\mathrm{al}^{14}$ performed a review of 52 cases of DDI taken from the literature and reported that endodontic therapy was attempted in only two cases. This paper reviewed two cases, of which one was reported as a case of DDII with relatively long roots, which was treated by conventional endodontic therapy; the other was a case of DDI which was treated surgically with 2-months follow-up.

Orthodontic treatment is suggested; however, further resorption of the roots, loosening of teeth, and premature exfoliation may occur due to the resistance of the short roots to the orthodontic forces. ${ }^{15}$

In the case of few teeth having enough root development, cast partial dentures can be a treatment option. Dental implants should be considered when growth is complete. Ridge augmentation procedures need to be carried out prior to implant placement in maxillomandibular alveolar atrophy due to early loss of teeth. ${ }^{3}$

\section{CONCLUSION}

The outcome of a prognosis of DD largely depends upon the age at which the diagnosis was made and the quality of the treatment provided. Early diagnosis helps in guiding variable measures to prolong the retention or rehabilitation of affected teeth to maintain good esthetics and function. The oral hygiene of the children must be established to have natural teeth as long as possible. Furthermore, it is appropriate that studies are performed to evaluate the prevalence of these types of dental anomalies.

\section{REFERENCES}

1. Ozer S, Ozden B, Otan Ozden F, Gunduz K. Dentinal dysplasia type I: a case report with a 6-year followup. Case Rep Dent 2013 May;2013:659084.
2. Witkop CJ Jr. Hereditary defects of dentin. Dent Clin North Am 1975 Jan;19(1):25-45.

3. Fulari SG, Tambake DP. Rootless teeth: dentin dysplasia type I. Contemp Clin Dent 2013 Oct-Dec;4(4):520-522.

4. Byahatti SM. Dentin dysplasia type I: a rare case report. Int J of Oral Health Sci 2013 Nov;3(1):57-60.

5. Sekerci AE, Etoz M, Sahman H, Sisman Y, Nazlim S. A rare condition affecting the primary and permanent dentition: Dentin dysplasia type I. J Oral Maxillofac Radiol 2013 May;1(1): 13-16.

6. Khandelwal S, Gupta D, Likhyani L. A case of dentin dysplasia with full mouth rehabilitation: a 3-year longitudinal study. Int J Clin Pediatr Dent 2014 May-Aug;7(2):119-124.

7. Wesley RK, Wysocki GP, Mintz SM, Jackson J. Dentin dysplasia type I: clinical, morphologic, and genetic studies of a case. Oral Surg Oral Med Oral Pathol 1976 Apr;41(4): 516-524.

8. Brenneise CV, Conway KR. Dentin dysplasia, type II: report of 2 new families and review of the literature. Oral Surg Oral Med Oral Pathol Oral Radiol Endod 1999 Jun;87(6): 752-755.

9. O'Carroll MK. The diagnosis of dentinal dysplasia Type I. Dentomaxillofac Radiol. 1994 Feb;23(1):52-53.

10. Azma E, Kia SJ, Nemati S. dentin dysplasia type I with hypomature amelogenesis imperfecta in an 18-year-old girl. J Dentomaxillofac Radiol Pathol Surg 2013 Autumn;2(3): 34-39.

11. Toomarian L, Mashhadiabbas F, Mirkarimi M, Mehrdad L. Dentin dysplasia type I: a case report and review of the literature. J Med Case Reports 2010 Jan;4:1.

12. Shokri A, Poorolajal J, Khajeh S, Faramarzi F, Kahnamoui HM. Prevalence of dental anomalies among 7- to 35-year-old people in Hamadan, Iran in 2012-2013 as observed using panoramic radiographs. Imaging Sci Dent 2014 Mar;44(1):7-13.

13. Ravanshad S, Khayat A. Endodontic therapy on a dentition exhibiting multiple periapical radiolucencies associated with dentinal dysplasia Type 1. Aust Endod J 2006 Apr;32(1): 40-42.

14. Ansari G, Reid JS. Dentinal dysplasia type I: review of the literature and report of a family. ASDC J Dent Child. 1997 Nov-Dec;64(6):429-434.

15. Scola SM, Watts PG. Dentinal dysplasia type I. A subclassification. Br J Orthod 1987 Jul;14(3):175-179. 